\title{
Flight Leader Concept for Wind Farm Load Counting: Offshore evaluation
}

\author{
T.S. Obdam \\ L.W.M.M. Rademakers \\ H. Braam
}

This report has been presented at the European Offshore Wind 2009 Conference, Stockholm, 14-16 September, 2009 


\title{
Flight Leader Concept for Wind Farm Load Counting: Offshore evaluation
}

\author{
T.S. Obdam, L.W.M.M. Rademakers, H. Braam \\ Energy Research Centre of the Netherlands (ECN) \\ Wind Energy \\ P.O. Box 1 \\ 1755 ZG Petten \\ Tel. (+31) 224564395 \\ Fax. (+31) 224568214 \\ obdam@ecn.nl
}

\begin{abstract}
ECN has developed a demo version of the Flight Leader model, which is based on a concept with which the accumulated mechanical loading of all turbines in an offshore wind farm can be estimated at acceptable costs. This information can be used to optimise and lower the cost of Operation \& Maintenance $(\mathrm{O} \& \mathrm{M})$, for example by prioritising inspections and replacements. In this paper the background and general concept of the Flight Leader model are presented. A full Flight Leader analysis has been performed using data from the Dutch offshore wind farm OWEZ. In this paper the approach and results of the analyses are discussed.
\end{abstract}

Keywords: Load Monitoring, Offshore Wind Energy, Operation \& Maintenance

\section{Introduction}

Operation \& Maintenance (O\&M) costs for offshore wind farms account for up to $30 \%$ of the $\mathrm{kWh}$ price [1]. Lowering these costs is an important prerequisite for the economical exploitation of large offshore wind farms. The adequate planning of condition based maintenance is one aspect that could decrease the O\&M costs; instead of having similar maintenance and inspection schemes for all turbines, the O\&M requirements for each turbine can be made more dependent on its accumulated mechanical loading [2].
The most obvious way to get insight in the loading of all turbines in an (offshore) wind farm is to instrument all turbines with load measurements on the critical components. However, in practice after a wind farm is built, the actual loads on components are measured in only very few occasions. This is mainly caused by the fact that an adequate measurement campaign is labour intensive, costly and time consuming, especially if all turbines need to be measured.

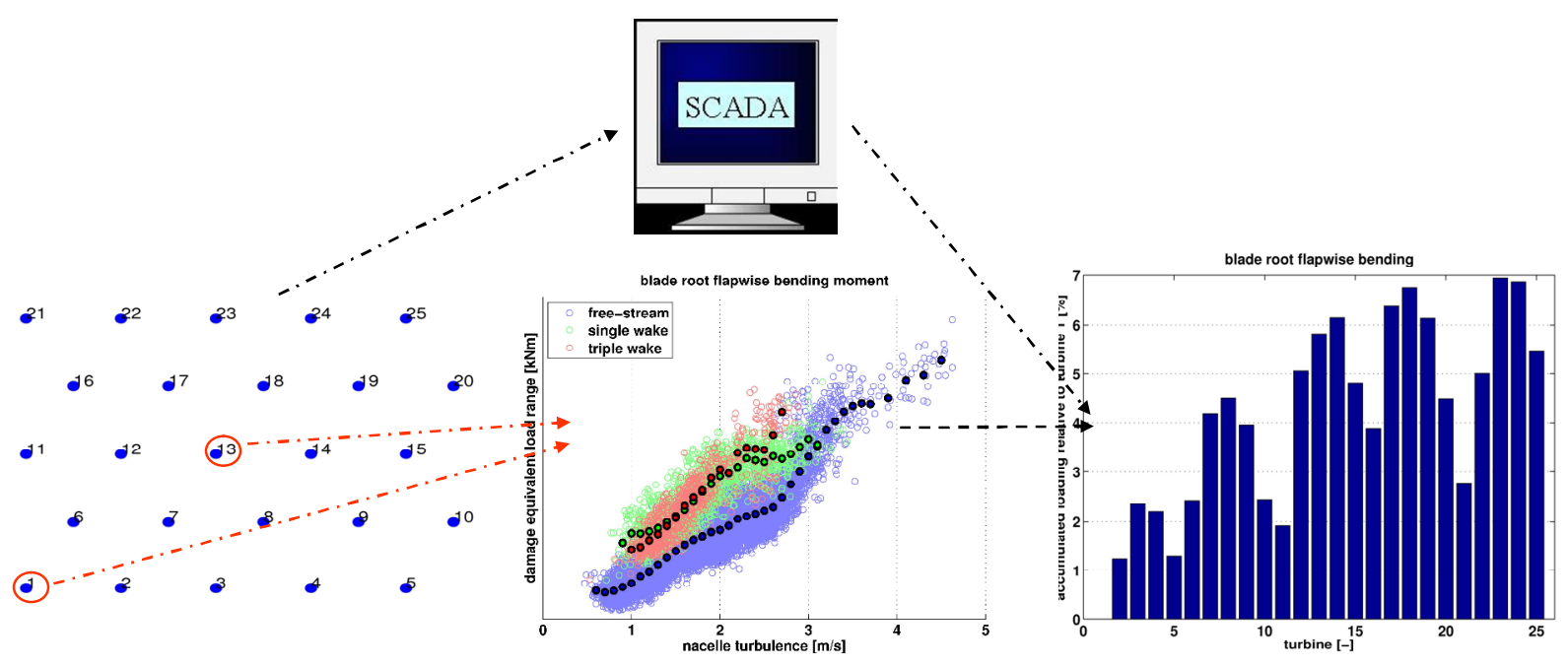

Figure 1: General structure of the flight leader computer model. 
ECN is developing the Flight Leader concept, which is a methodology where only a small number of turbines at strategic locations in the offshore wind farm are equipped with mechanical load measurements. Using the measurements at these so-called 'Flight Leader' turbines relations are established between standard (SCADA) signals and load indicators. Combining these relations with the standard signals of all other turbines in the wind farm, offers the possibility to keep track of the accumulated mechanical loading of all turbines in the offshore wind farm at low costs. This is illustrated in Figure 1.

ECN has developed a demo version of a software model [3,4], which includes all aspects of the Flight Leader concept. The software is intended to be used by operators of offshore wind farms and is used to process the SCADA data and mechanical load measurements from the offshore wind farm. The main output of the model is a comparison of the accumulated mechanical loading of all turbines in the offshore wind farm. This information can subsequently be used to optimise O\&M strategies, for example by prioritising the inspection or replacement of certain components on the heavier loaded turbines. The structure and functionality of the software model is explained in more detail in chapter 2 .
Key to the application of the Flight Leader concept are the relations between standard (SCADA) signals and load indicators. The more accurate these relations, the more reliable are the calculations of accumulated loading. Previously $[3,4]$, it has been proven that artificial neural networks can be applied for accurately estimating load indicators using only 10-minute statistics of standard SCADA parameters. First a short introduction on neural networks is given in chapter 3 . In chapter 4 the approach and results for the evaluation of the Flight Leader are presented. Finally, in chapter 4 the results of the Flight Leader software, implemented at an offshore wind farm, are presented.

\section{The flight leader model}

In this chapter the structure of the flight leader model will be treated in more detail. The general structure for the flight leader computer model is shown in the flowchart in Figure 2.

In the following subsections the different parts of the flight leader model will be discussed in more detail.

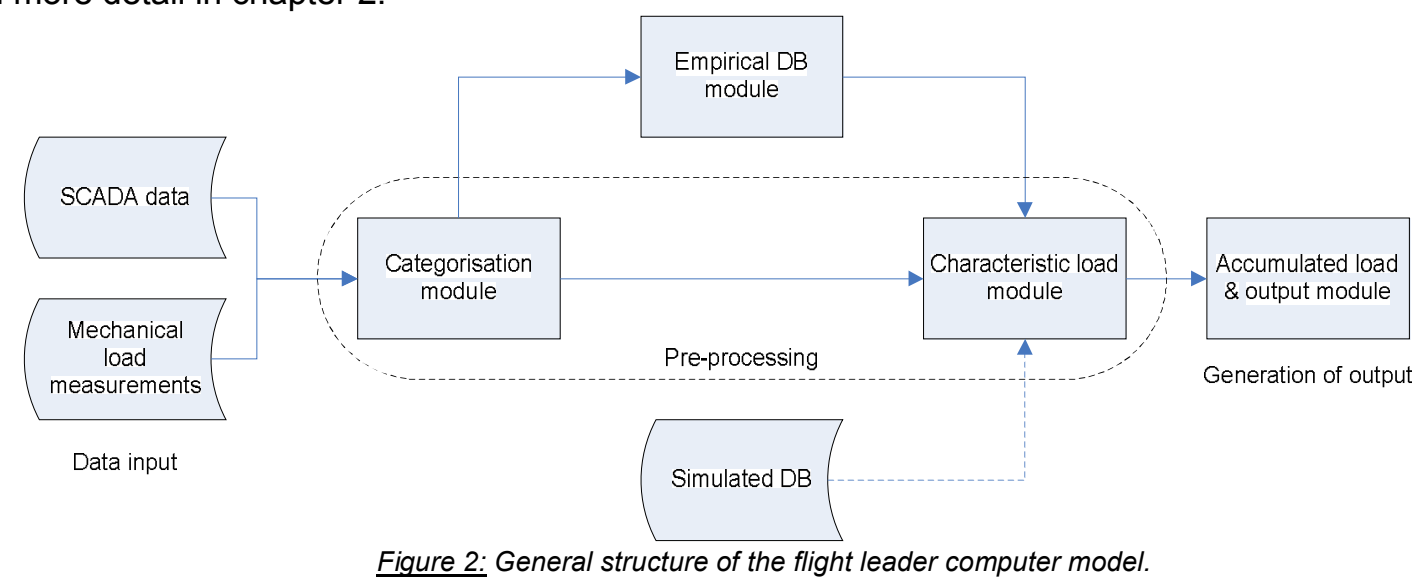

\subsection{Data input}

The input for the flight leader model is the data that are collected from the offshore wind farm. Two types of data can be distinguished; (1) SCADA data, which are being collected from all turbines and (2) mechanical load measurements, which are being collected only from the flight leader turbines. Both data should be collected as 10-minute statistics.

\subsection{Data categorisation}

Unfortunately a wind turbine does not always operate in normal power production mode.
Furthermore, when located in an (offshore) wind farm, wind turbines do not always experience free-stream wind conditions. Both mentioned conditions are expected to have an effect on the mechanical loading.

In order to take this into account the first step of the flight leader model is to categorise each 10-minute timestamp in the dataset in one of the possible combinations of the three predefined turbine states $j$ and wake conditions $k{ }^{1}$

\footnotetext{
${ }^{1}$ It is assumed that wake conditions are only relevant in case a wind turbine operates in normal power production.
} 
The possible combinations are indicated in Table 1

Table 1: Possible combinations of turbine states \& transitional modes and wake conditions.

\begin{tabular}{|c|c|c|}
\hline ID & $\begin{array}{c}\text { Turbine state or } \\
\text { transitional mode } j\end{array}$ & Wake condition $k$ \\
\hline 1.1 & \multirow{2}{*}{$\begin{array}{c}\text { Normal power } \\
\text { production }\end{array}$} & Free-stream \\
1.2 & & Partial wake \\
\hline 1.3 & & Full wake \\
\hline 2.1 & Parked/ldling & Not applicable \\
\hline 3.1 & Transient events & \\
\hline
\end{tabular}

\subsection{Empirical database}

After all available data have been categorised the measurements from the Flight Leader turbines can be used to establish relations between (standard) SCADA parameters and load indicators, which are representative for the damage, aging or degradation of a certain component.

As mentioned in the previous section, these relations are expected to differ for the identified turbine states \& transitional modes and wake conditions. Therefore the relations between SCADA parameters and load indicators have to be determined for each of the possible combinations shown in Table 1.

The software model offers the possibility to characterise the relations using more traditional methods such as interpolation or multivariate regression but also using artificial neural network techniques.

\subsection{Simulation database}

In the period directly after the commissioning of the offshore wind farm little measured data are available. Therefore it might be beneficial to incorporate the results of aero-elastic simulations into the flight leader model. This is particularly interesting for those situations with a low probability of occurrence, such as emergency shutdowns or extremely high wind speeds.

\subsection{Estimating load indicators}

Next step is estimating the load indicators at all turbines in the offshore wind farm. This is achieved by combining the SCADA data, collected at all turbines, with the relations between SCADA parameters and load indicators as stored in the empirical database. Optionally, for this process also results from aero-elastic simulations can be incorporated.

The situation might occur that for a certain turbine for a certain amount of time no SCADA data are available. For these periods the load indicators cannot be estimated neither with the empirical nor the simulation database. In order to ensure a fair comparison of the total accumulated loading the software also contains a procedure for handling missing data.

\subsection{Output}

Finally, the last part of the model is the process of generating and displaying the desired output of the flight leader model. The main output consists of a comparison of the accumulated mechanical loading of all turbines in the offshore wind farm. This output needs to be shown for the several load indicators (e.g. blade root bending, tower bottom bending or main shaft torque).

Besides the main output the software model can calculate and display various breakdowns of the accumulated loading. For instance the contribution of each turbine state or transitional mode or wake condition to the total accumulated loading can be displayed. Furthermore the load accumulation per time period can be studied. These outputs can be used to get more insight in the performance of the offshore wind farm and what operating conditions have the largest impact on the loading of the turbines in the offshore wind farm.

\section{Artificial neural networks}

Besides the more 'classical' techniques of interpolation and regression so-called 'artificial neural networks' can also be applied to model the relationship between two or more variables $[5,6]$

\subsection{General description}

A neural network in fact represents a mathematical model, where a number of (transfer) functions are connected in parallel and, possibly, also in series. Based on the weighted sum of multiple input signals each transfer function calculates a value, which subsequently serves as input for the next transfer function. The transfer function, including the weighted summation of multiple input signals, is labelled as neuron. A neural network with a sufficient number of neurons is, in theory, able to approximate every possible function.

A schematic representation of a neuron and a neural network (consisting of two 'hidden' layers of neurons) is shown in Figure 3. 

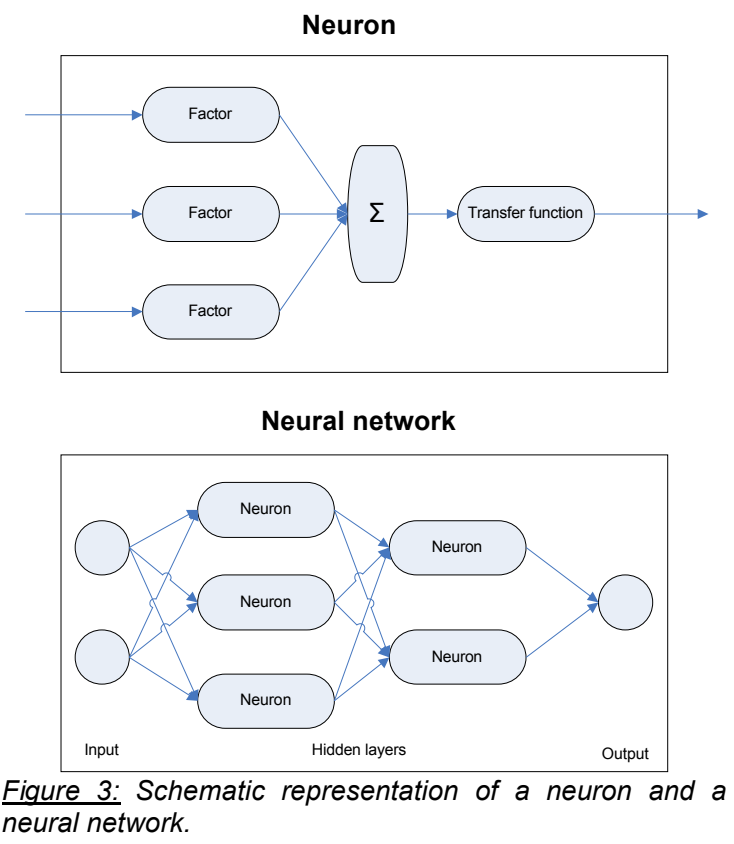

\subsection{Application}

For the analyses that will be described in chapter 4 the MATLAB ${ }^{\circledR}$ Neural Network Toolbox ${ }^{\mathrm{TM}}$ has been used. The neural networks are trained using the Levenberg-Marquardt backprogagation algorithm. In order to prevent overfitting the early stopping technique is used.

\section{Evaluation of the Flight Leader at OWEZ}

In this chapter the approach and results of the evaluation of the Flight Leader software at the Offshore Wind farm Egmond aan Zee (OWEZ) are presented. In the first two subsections some information of the wind farm and the dataset is given. In the following subsections the results from all steps in the evaluation process are discussed.

\subsection{The OWEZ wind farm}

OWEZ is the first Dutch offshore wind farm, located 10-18 km from the village Egmond aan Zee. The farm consists of 36 Vestas V90 turbines, which are pitch-controlled variablespeed machines with a rated power output of 3 MW.

Two turbines in the farm are equipped with mechanical load measurements on blades and tower and will therefore act as Flight Leader turbines.

\subsection{Dataset}

The evaluation has been performed using 9 months of measured data from all 36 turbines. Available SCADA signals include nacelle wind speed, rotor rotational speed, pitch angle, elec- trical power output and nacelle yaw direction. Mechanical load measurements are performed on blade (flapwise and edgewise) and tower (north-south and east-west). Additionally, data from the meteorological mast and nearby wave buoy are available.

\subsection{Data categorisation}

As discussed in chapter 2 the first step of the analysis is to categorise the data for each turbine $i$ and 10-minute timestamp $t$ in one of the pre-defined load cases (see Table 1).

\subsection{Selection of load indicator and SCADA parameters}

The evaluation of the Flight Leader software has been performed for two load indicators ${ }^{2}$; the $1 \mathrm{~Hz}$ damage equivalent load range $\Delta F_{E Q}$ of:

- Blade root flapwise bending;

- Tower bottom for-aft bending;

The damage equivalent load range $\Delta F_{E Q}$ is the load range that for some arbitrarily chosen number of cycles $N$ would, in theory, produce the same damage as all actual load ranges (which follow from rain flow counting) combined:

$$
\Delta F_{E Q}=\sqrt[m]{\frac{\sum_{i} n_{i} \cdot \Delta F_{i}^{m}}{N}}
$$

where $m$ is the Wöhler coefficient, $n_{i}$ the actual number of cycles and $\Delta F_{i}$ the actual load range for each occurring case $i$.

A trial-and-error approach has been adopted in order to assess which signals should be inand excluded in the artificial network. It has also been chosen to, at first, not to include any signals from the meteorological mast or wave buoy. The selected SCADA parameters are listed in Table 2 for each load case (for both load indicators the same set of SCADA parameters has been used).

Table 2: SCADA signals used to estimate the load indicator for tower for-aft bending for each load case.

\begin{tabular}{c|cc|cc|cc|cc}
\hline $\begin{array}{c}\text { Load } \\
\text { case }\end{array}$ & \multicolumn{2}{|c|}{$\begin{array}{c}\text { Wind } \\
\text { speed }\end{array}$} & \multicolumn{2}{|c|}{$\begin{array}{c}\text { Rotor } \\
\text { speed }\end{array}$} & \multicolumn{2}{c|}{$\begin{array}{c}\text { Pitch } \\
\text { angle }\end{array}$} & \multicolumn{2}{|c|}{ Power } \\
\hline$I D$ & avg & std & avg & std & avg & std & avg & std \\
\hline 1.1 & & & 0 & 0 & 0 & 0 & 0 & 0 \\
1.2 & & & 0 & 0 & 0 & 0 & 0 & 0 \\
1.3 & & & 0 & 0 & 0 & 0 & 0 & 0 \\
2.1 & 0 & & 0 & & 0 & & & \\
3.1 & 0 & 0 & 0 & 0 & 0 & 0 & 0 & 0 \\
\hline
\end{tabular}

\footnotetext{
2 For the sake of compactness only the results for tower for-aft bending are presented in this paper.
} 


\subsection{Relation SCADA parameters and load indicator}

After identifying the load indicators and relevant SCADA parameters the next step is to establish the relation between the selected SCADA signals and the load indicator. A separate relation has to be devised for each of the five identified load cases. The relations are characterised by an artificial neural network, which is trained using data from both Flight Leader turbines.

Half of the total amount of data is used for training the network. Another $25 \%$ is used to validate the network's performance for every iteration step and to halt training at the point where generalisation starts decreasing. The final $25 \%$ is used as an independent measure (has no influence on the network's training process) of the network's performance when fed with new data.

The performance of the trained artificial neural network for the load case power production \& free-stream conditions is shown in Figure 4.

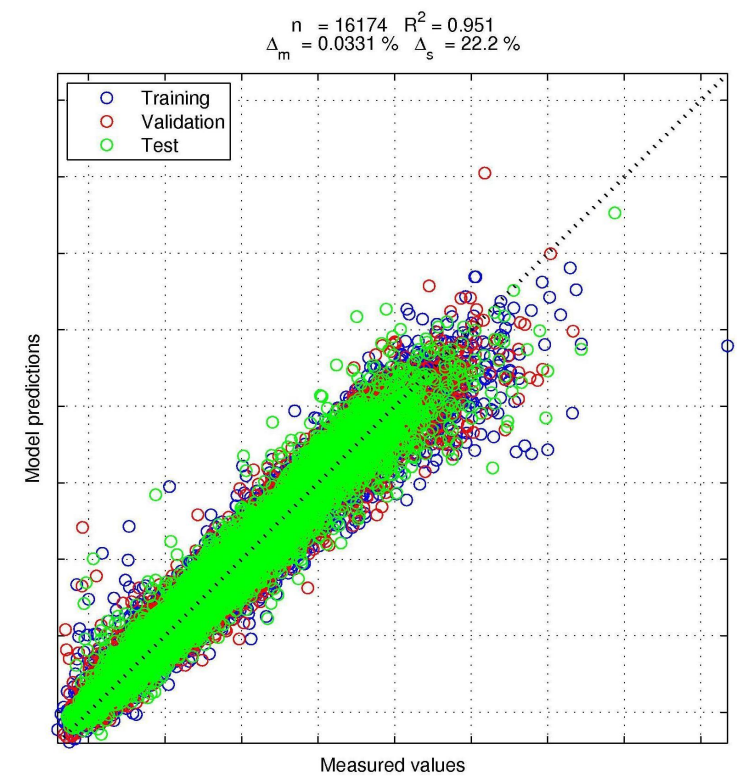

Figure 4: Performance of the neural network trained for estimating the tower bottom for-aft bending load indicator for a turbine in power production under free-stream conditions..

The results presented in the figure indicate that a good relation exists between the selected SCADA parameters (see Table 2) and the load indicator for the tower bottom for-aft bending moment (see equation 1). This is confirmed by the value of the coefficient of determination $\left(R^{2}\right.$ $=0.95$ ). When studying the green data points, which represent the 'test' dataset (which has had no influence on the network's training), it can be seen that here also a good performance is achieved. This is an important indicator for the generalisability of the neural network (its ability to make accurate predictions when fed with new data). Therefore it can be expected that the trained neural network will also make accurate predictions for the other turbines in the same wind farm for this load indicator.

The results (number of data points and coefficient of determination) for all five load cases are summarised in Table 3.

Table 3: Number of data points and coefficient of determination for the established relations between SCADA signals and the load indicator for tower for-aft bending.

\begin{tabular}{c|cc}
\hline Load case & Data points & $R^{2}$ \\
\hline 1.1 & 16174 & 0.9507 \\
1.2 & 3266 & 0.9439 \\
1.3 & 1669 & 0.9593 \\
2.1 & 12987 & 0.6812 \\
3.1 & 1193 & 0.9530 \\
\hline
\end{tabular}

The table indicates that for all load cases but parked/idling a good accuracy (of around $R^{2}=$ 0.95 ) is achieved. This might be unexpected, since no wave-describing parameters are included as independent variables in the artificial neural network (see Table 2). However, wave height and direction are, in general, strongly correlated with wind speed and direction. As a result the fluctuations in the tower bottom foraft bending for an offshore turbine can be accurately estimated without information on the wave conditions.

The main reason why for parked/idling more scatter is observed is the fact that no wavedescribing SCADA signals are included as independent variables in the relation. When the turbine is not in operation it also generates no thrust force, which means that the fluctuations in the tower bottom for-aft bending are solely caused by wave-induced loading. In case the 10-minute significant wave height, direction and period are included as independent parameters a significantly improved accuracy is achieved $\left(R^{2} \approx 0.80-0.85\right)$. However, since the data from the wave buoy is missing for large chunks of time, only about 3000 data points are available. Including these parameters in the relation also has the consequence that the Flight Leader software cannot make any predictions of the value of the load indicator for the periods where no wave data are available. This would lead to a significant error when estimating the total load accumulation and therefore it is decided to establish the

\footnotetext{
${ }^{3}$ Note that the shown numbers for data points and coefficient of determination represent the 'training' dataset. The 'training' dataset represents $50 \%$ of the total amount of data available.
} 
relations without including wave-describing parameters.

\subsection{Load indicator estimation}

In the previous section it has been described that for each of the five load cases a relation between the selected SCADA signals and load indicator has been determined and stored in the empirical database of the Flight Leader software. Next step is to combine these relations with the SCADA collected from all 36 turbines in the offshore wind farm in order to make an estimate of the value of the load indicator the tower for-aft bottom bending moment for each turbine $i$ and timestamp $t$.

After the load estimation has been executed two post-processing procedures are performed. Firstly, outliers are identified using the criteria that the calculated value of a load indicator can never be smaller or larger than a certain factor $\beta$ multiplied with, respectively, the minimum and maximum measured value of the load indicator. Timestamps $t$ which do not meet this criteria have been classified as $\mathrm{NaN}$ in the dataset.

$$
\begin{aligned}
& (1-\beta) \cdot \min \left(\Delta F_{E Q, i F L}\right) \\
& \leq \Delta F_{E Q, i} \leq \\
& (\beta+1) \cdot \max \left(\Delta F_{E Q, i F L}\right)
\end{aligned}
$$

The initial analysis has been performed for different values of $\beta$. However, it was found that this did not have a significant influence on the number of corrected outliers. The analyses described in this report have been performed using $\beta=0$ (no extrapolation possible).

Next step is to ensure that for all turbines an equal amount of data is available. If for a certain turbine $i$ at a certain timestamp $t$ the value of the load indicator is unknown the value will be estimated by taking the average value of load indicator $c$ at all turbines in the farm for which at timestamp $t$ the value of load indicator $c$ is known.

$$
\Delta F_{E Q, c, i_{\text {unavailab }}, t}=\frac{1}{n} \cdot \sum_{i_{\text {avaiable }}}^{n} \Delta F_{E Q, c, i_{\text {available }}, t}
$$

where $\Delta F_{E Q, c, t}$ is the value for characteristic load $c$ at timestamp $t$, $i_{\text {available }}$ and $i_{\text {unavailable }}$ represent turbine $i$ for which SCADA data are, respectively, available and unavailable, and $n$ is the number of turbines for which the value of load indicator $c$ is known.
The results of the post-processing are shown in Figure 5. The top graph indicates the number of data points classified as outliers, the middle graph shows the amount of corrected data for each turbine and the bottom graph indicates the percentage of missing data after all post-processing steps have been performed.
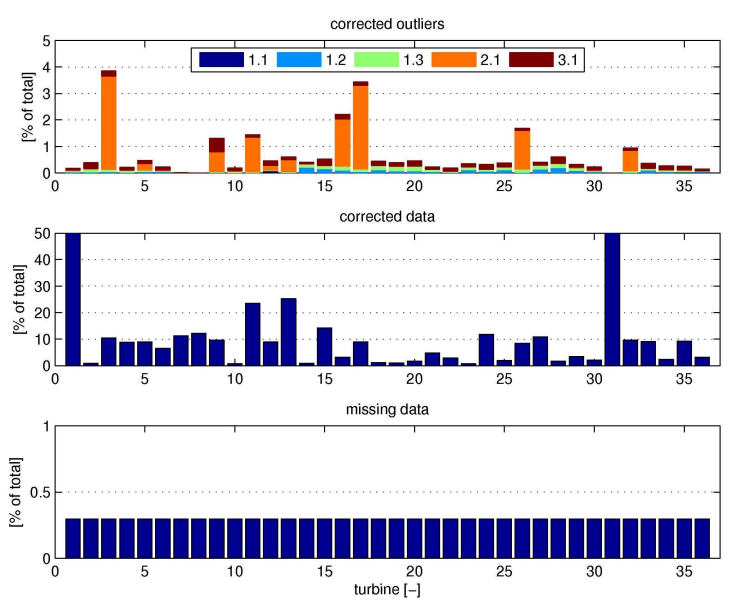

Fiqure 5: Results of the post-processing steps for estimating the values of the load indicator for tower for-aft bending.

It can be seen that for most turbines the number of outliers is quite low (less than $0.4 \%$ ) for all turbines. However, for some turbines about up to $4 \%$ of the estimated values of the load indicator have been classified as outlier. It can also be observed that for these turbines most outliers occur for the load case parked/idling. As can be seen in Table 3 this is also the load case where a less accurate relationship between the selected SCADA parameters and load indicator has been established.

Furthermore, the results of post-processing indicate that the amount of corrected data varies greatly over the different turbines. For a number of turbines only about $2 \%$ of all load estimations had to be done using data from other turbines. Subsequently also a number of turbines has had around $10 \%$ of their load estimations corrected. Finally, for four turbines more than $20 \%$ of the estimated values of the load indicator have been derived from other turbines. This should be considered when comparing the load accumulation of these turbines with the other turbines as will be described further on in this report.

The bottom graph indicates that after both post-processing steps have been completed the amount of missing data is identical for all 36 turbines and is equal to less than $0.5 \%$ of the total amount of data. 


\subsection{Output}

Now the values of the load indicator for tower bottom for-aft bending have been estimated for each turbines $i$ for each 10-minutetime period $t$ it is possible to calculate the total load accumulation for each turbine $i$. Total load accumulation $\Delta S_{\text {total, } i}$ for each turbine $i$ is calculated as follows:

$\Delta S_{\text {total }, i}=\sqrt[m]{\sum_{t=1}^{N} \Delta S_{i, t}^{m}}$

where $\Delta S_{i, t}$ is the value of the load indicator for turbine $i$ and 10-minute timestamp $t$.

Subsequently, the relative difference in load accumulation is calculated according:

Difference $=\frac{\Delta S_{\text {total }, i}-\Delta S_{\text {total, iref }}}{\Delta S_{\text {total, } \text {, iref }}}$

where $\Delta S_{\text {total, } i}$ and $\Delta S_{\text {total,iref }}$ are the total load accumulation for turbine $i$ and reference turbine $i_{\text {ref }}$ respectively.

In Figure 6 a comparison of the load accumulation for the tower bottom for-aft bending moment is shown. The load accumulation of each turbine $i$ is shown relative to the load accumulation of turbine 18.

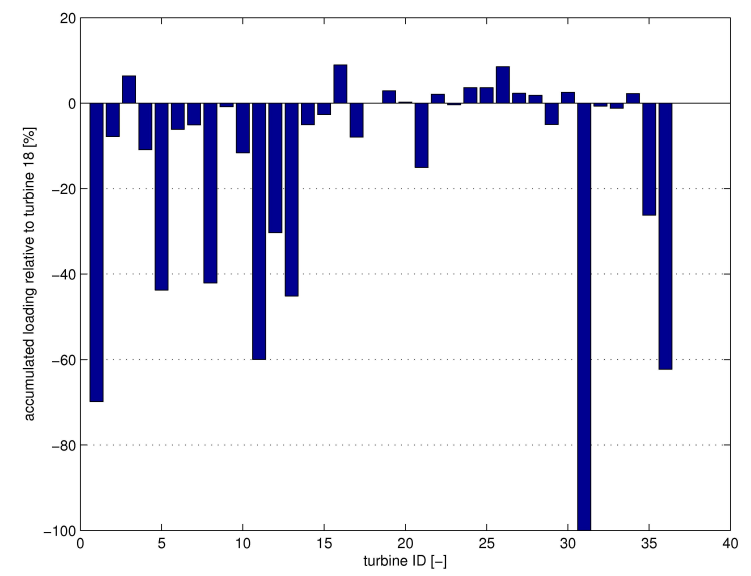

Figure 6: Load accumulation of all five turbines relative to load accumulation of turbine 18.

The figure indicates that most turbines have suffered a load accumulation which is roughly within about $10 \%$ of the load accumulation of turbine 18. However, also about 10 turbines have accumulated significantly less load. When interpreting these results the outcome of the load estimation post-processing steps should be kept in mind (see Figure 5). It was shown that for turbines 1, 11, 13 and 31 more than $20 \%$ of all estimated values of the load indicator have been derived by averaging the data from the other turbines. This procedure is necessary to ensure that for all turbines an identical amount of data is available for calculating load accumulation but also leads to an inaccurate calculation of load accumulation for the mentioned turbines.

In order to get more insight in the presented results a breakdown of the total load accumulation has been calculated (see Figure 7). The graph shows the contribution of three of the five load cases to the total load accumulation (blue bars). In order to interpret the results in both graphs the amount of data is also illustrated (red bars). Data from all 36 turbines have been used.

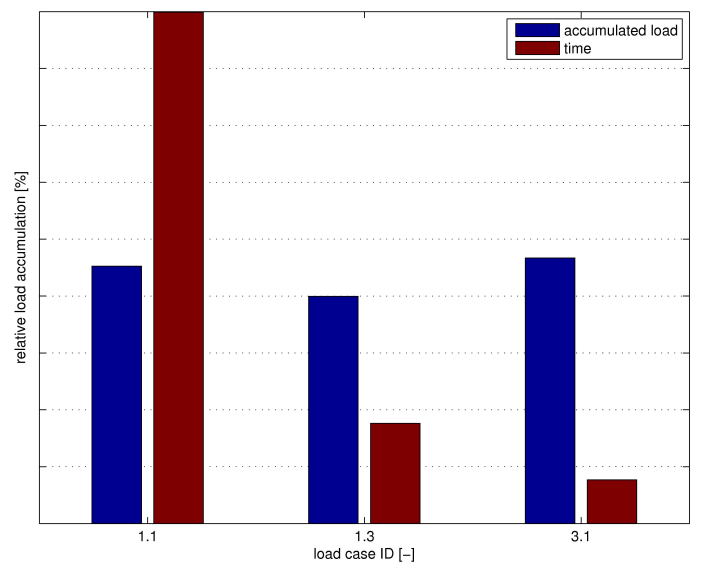

Fiqure 7: Breakdowns of the load accumulation per load case. Note that the results of only three of the five load cases are displayed.

The graph shows that on average the 36 turbines operate in power production and under free-stream conditions for most of the time. However, the total load accumulation for this load case is a much smaller part of the total. The opposite is found in case the turbine operates in wake conditions. Especially if the turbines are facing full wake conditions the load accumulation is more than two times as large compared to the amount of time the turbines operate in these conditions. Although, not shown in the figure it has been found that when the turbines are in parked or idling condition still a significant amount of load accumulation occurs. For onshore turbines this was not the case [6], which indicates that for this load case the wave-induced loading is dominant. Finally, relatively the largest load accumulation occurs during transient events. This load case accounts for a very small part of the total data but still the load accumulation during this load case is similar to the one for power production under free-stream conditions for which a staggering 10 times as many data are available. 


\subsection{Validation}

Last step in the analysis is the validation of the accuracy of the Flight Leader predictions. In order to do this the predicted and measured load accumulation for each load case are calculated for both Flight Leader turbines. The prediction errors are subsequently calculated using:

$$
\text { Error }=\frac{\Delta S_{\text {total,pred }}-\Delta S_{\text {total,meas }}}{\Delta S_{\text {total,meas }}}
$$

where $E_{\text {total,pred }}$ and $E_{\text {total,pred }}$ are the predicted and measured total electricity production subsequently.

The resulting prediction errors are shown in Table 4.

Table 4: Prediction errors of the Flight Leader software for the load indicator for tower bottom for-aft bending.

\begin{tabular}{c|cc}
\hline Load case & FL turbine 1 & FL turbine 2 \\
\hline 1.1 & $-1.25 \%$ & $-0.16 \%$ \\
1.2 & $-0.77 \%$ & $-1.36 \%$ \\
1.3 & $-0.88 \%$ & $-1.93 \%$ \\
2.1 & $-7.88 \%$ & $-6.60 \%$ \\
3.1 & $-1.65 \%$ & $-0.15 \%$ \\
\hline
\end{tabular}

The results in the table show that the Flight Leader predictions of load accumulation are very accurate. For the power production and transient event load cases the prediction errors are smaller than $2 \%$. For the parked/idling load case the errors are slightly larger, which can be contributed to the fact that for this load case the relation between SCADA parameters and load indicator showed significantly more scatter compared to the relation for the other load cases.

\subsection{Conclusions}

A full Flight Leader analysis has been performed using data from the OWEZ offshore wind farm. The analysis has been performed for the tower bottom for-aft bending load. For this load the damage equivalent load range is used as load indicator.

A trial-and-error approach has been adopted in order to determine what SCADA parameters are relevant for estimating the values of the load indicator. For each of the five defined load cases a separate relation between the selected SCADA signals and the values of the load indicator has been determined. For almost all load cases an accurate relation is established. Only for the parked/idling load case the relation is surrounded by more scatter, which is caused by the fact that no wave- describing parameters are included in the relation.

After establishing all required relations the values of the load indicators are estimated for all 36 turbines in the offshore wind farm. Subsequently, the 10-minute load indicator values have been summed in order to calculate total load accumulation of each turbine in the farm. After comparing the total load accumulation of all turbines it has been found that the difference in total load accumulation is smaller than around $10 \%$ for most of turbines. A few exceptions exist, mainly for the turbines that have been in parked of idling state for large periods of time.

It has also been analysed what load cases contribute most to total load accumulation. The most striking observation is the very high contribution of transient events to the total load accumulation. Despite its low frequency of occurrence for the load accumulation during this load case is about equal to the load accumulation during power production in freestream conditions. Furthermore, also load accumulation during wake operation is relatively high. In contradiction to what has been observed onshore, during the parked/idling load case still significant load accumulation occurs, which can be attributed to wave-induced loading.

Finally, also the output of the Flight Leader software has been validated by comparing the predicted and measured total load accumulation for both Flight Leader turbines. Very small prediction errors were found for all load cases but parked/idling. The lower accuracy of the Flight Leader software here can be explained by the lower accuracy of the relation between SCADA parameters and load indicator for this load case.

\section{Status and future work}

Functional and technical specifications for the Flight Leader software model have been completed. On the basis on these detailed specifications a demo version of the Flight Leader software model has been programmed in MATLAB ${ }^{\circledR}$.

The demo software model has been evaluated extensively using data from both ECN's EWTW wind farm and Dutch offshore wind farm OWEZ. Based on the findings on the performed analyses the software has been adjusted where necessary. Furthermore an inhouse review of the software is currently being performed. 
Furthermore, results of aero-elastic simulations will be incorporated in the Flight Leader software with the target of (1) comparing the empirical and simulated relations between SCADA parameters and load indicator, and (2) to ensure that sufficient data is available for load cases for which little empirical data is available (e.g. emergency shutdowns).

\section{Acknowledgements}

This study is carried out and co-financed by the Bsik programme 'Large-scale Wind Power Generation Offshore' of the consortium We@Sea (http://www.we-at-sea.org).

The Bouw Combinatie Egmond (BCE) consortium is acknowledged for making data from the OWEZ wind farm available for further evaluation and improvement of the Flight Leader concept and software.

\section{References}

[1] L.W.M.M. Rademakers, H. Braam, M.B. Zaaijer, G.J.W. van Bussel; Assessment and Optimisation of Operation and Maintenance of Offshore Wind Turbines; Proceedings European Wind Energy Conference 2003.

[2] L.W.M.M. Rademakers, H. Braam, T.S. Obdam; Estimating Costs of Operation \& Maintenance for Offshore Wind Farms; ECN-M--08-027; Presented at the European Wind Energy Conference 2008, Brussels.

[3] T.S. Obdam, L.W.M.M. Rademakers, H. Braam; Flight Leader Concept for Wind Farm Load Counting and Performance Assessment; ECN-M--09-054; Presented at the European Wind Energy Conference 2009, Marseille.

[4] T.S. Obdam, L.W.M.M. Rademakers, H. Braam; Flight Leader Concept for Wind Farm Load Counting: First Offshore Implementation; ECN-M--09-114; Presented at the OWEMES Conference 2009 Brindisi.

[5] S. Schwarz, R. Dittmar, A. Anders; Determination of Actual Fatigue Loading on Wind Turbines Based on Neural Networks; 2005.

[6] N. Cosack, M. Kühn; Prognose von Ermüdungslasten an Windenergieanlagen mittels Standardsignalen und neuronaler Netze; Presented at the Dresdner Maschinenelemente Kolloquium, Dresden; December 2007. 